\title{
Endoscopically-Assisted Percutaneous Unilateral Atlantoaxial Screw-Rod Nonfusion Fixation Treatment for Type II Odontoid Fractures in Geriatric Patients: Case Series and Technical Note
} Lei Shi, MD, PhD ${ }^{1,2}$, Rui Deng, MD, PhD ${ }^{1,2}$, Qing-Yan Long $\mathrm{BS}^{3}$, Lei Chu, MD, $\mathrm{PhD}^{1,2}$,
Ke-Xiao Yu, MD, PhD ${ }^{1,2}$, Zhou Xu, MD, PhD ${ }^{1}$, and Zhong-Liang Deng, MD, PhD ${ }^{1,2}$

From: 'Department of Orthopedics, the Second Affiliated Hospital of Chongqing Medical University, Chongqing, China; ${ }^{2}$ Geriatric Clinical Research Center of Chongqing, Chongqing, China; ${ }^{3}$ Clinical Skills Training Center, the Second Affiliated Hospital of Chongqing Medical University, Chongqing, China

Address Correspondence: Zhong-Liang Deng, MD, PhD Department of Orthopedics, the Second Affiliated Hospital, Chongqing Medical University, No. 76 Linjiang Road Yuzhong District, Chongqing, 400010, PR China

Email: zhongliang.deng@yahoo.com; zhongliang.deng@qq.com

Disclaimer: Funding: The study was supported by the Special Foundation for Social Safeguard and Scientific Innovation of

Chongqing (No. cstc2016shmsztzx10001-6), Chongqing Research and Innovation project of graduate students (No. YB17112), and the key project of Chongqing Science and Technology Commission(No. cstc2019jscx-gksbool4), and the key project of Chongqing Health Commission(No.2016ZDXMoo7.

Conflict of interest: Each author certifies that he or she, or a member

of his or her immediate family, has no commercial association (i.e., consultancies, stock ownership, equity interest, patent/licensing arrangements, etc.) that might pose a conflict of interest in connection with the submitted manuscript.

Manuscript received: 03-19-2019 Accepted for publication: 07-09-2019

Free full manuscript: www.painphysicianjournal.com
Background: Considerable controversy exists regarding the optimal treatment for type II odontoid fractures in geriatric patients. Surgical intervention can help patients return to their prior level of function as rapidly as possible while avoiding the morbidity and mortality associated with prolonged and bedbound hospitalization. However, the optimal treatment is still a difficult choice for patients with increased risk from anesthesia.

Objectives: The objective of our study was to describe an innovative method of endoscopically-assisted percutaneous unilateral C1 lateral mass screw and C2 pedicle screwrod nonfusion fixation for type II odontoid fractures in geriatric patients.

Study Design: A case series design and technical notes

Setting: This study took place at Second Affiliated Hospital of Chongqing Medical University.

Methods: Seven geriatric patients (> 65 years) with type II odontoid fractures and an American Society of Anesthesiologists (ASA) score of 2 or higher received endoscopicallyassisted percutaneous unilateral atlantoaxial screw-rod nonfusion fixation. After surgery, all patients were required to wear a rigid collar full-time for 12 weeks. Intraoperative data, the bone union time, American Spinal Injury Association (ASIA) scale scores, Neck Disability Index (NDI) scores, and postoperative complications were collected for assessment.

Results The surgical goal was successfully achieved in all patients, 3 of whom had high ASA scores $(\geq 3)$ and underwent surgery under local anesthesia. The operative time ranged from 112 to 169 minutes (mean, 131.1 minutes). No neurovascular complications were observed intraoperatively or postoperatively. All patients rapidly returned to their prior level of function and were followed up for 12 to 24 months (average: 16.9 months). Bone union was achieved in all patients.

Limitations: This study is limited by being a retrospective study.

Conclusions: Endoscopically-assisted percutaneous unilateral atlantoaxial screw-rod nonfusion fixation is a feasible technique for type II odontoid fractures in geriatric patients. This method offers a compromise between non-operative and operative treatment and allows geriatric patients to rapidly return to their prior level of function.

Key words: Endoscopically-assisted surgery; geriatric patient; percutaneous atlantoaxial fixation; type II odontoid fracture; unilateral nonfusion fixation

Pain Physician 2020: 23:E241-E250 
() dontoid fracture is the most common individual cervical spine fracture in patients $>65$ years of age (1-3). These fractures are commonly classified into 3 types according to the Anderson and D'Alonzo classification (4). Type II fracture is the most common fracture pattern of odontoid injuries in $82 \%$ to $90 \%$ of geriatric patients $(2,5)$. This injury generally results from low-energy impacts, such as falls, in the geriatric population $(5,6)$.

In recent years, the optimal treatment for type II geriatric odontoid fractures has remained controversial. Non-operative management in geriatric patients is associated with reported high nonunion rates of $21 \%$ to $62.5 \%(6-8)$. Geriatric patients are at a higher risk of nonunion due to a combination of osteoporosis, watershed blood supply, and high anatomic strain (2). Much of the morbidity associated with type II odontoid fractures has generally been considered to be due to the risk of nonunion and ensuing instability and neurologic injury (9). However, recent literature shows a significant decrease in mortality rates and an increase in positive outcomes with surgical intervention in geriatric patients with type II odontoid fractures $(1,7,10,11)$.

Optimal treatment of geriatric patients with a type II odontoid fracture would help them return to their prior level of function as rapidly as possible while avoiding the morbidity and mortality associated with prolonged and bedbound hospitalization (7). Additionally, individual factors and associated comorbidities among geriatric patients and the safety of general anesthesia may require comprehensive consideration before any attempt at surgical treatment (10). An increased American Society of Anesthesiologists (ASA) score reportedly predicted significant increases in complication rates for morbidity and mortality postoperatively (12). Consequently, an optimal treatment that avoids the disadvantages of non-operative management and decreases the surgical risk is needed for patients with increased risk from anesthesia.

\section{Methods}

From July 2016 to July 2018, 7 geriatric patients who suffered type II odontoid fractures (Anderson and D'Alonzo classification) received endoscopicallyassisted (Spinendos, Munchen, Germany) percutaneous unilateral atlantoaxial screw-rod nonfusion fixation treatment. The inclusion criteria were: (a) type II odontoid fractures with an intact transverse atlantal ligament, (b) fracture displacement that could be reduced by preoperative traction, and (c) an age greater than
65 years and an ASA score of 2 or higher. Five female patients and 2 male patients with a mean age of 73.1 years (range: $66-89$ years) were included. The patient characteristics are presented in Table 1. Parameters including operative time, blood loss volume, surgical complications, and fracture union time were collected for assessment.

\section{Preoperative Preparation}

All patients underwent computed tomography (CT) angiography of the cervical spine to preoperatively detect the occurrence of vertebral artery (VA) variation. The optimal entry point, screw path, and diameters and lengths of the screws were determined by multiplanar CT reconstruction. Cervical magnetic resonance imaging (MRI) was required when patients presented a spinal cord injury or when soft tissue damage assessment was needed.

\section{Surgical Procedures}

All surgeries were performed by a senior spine surgeon. Three of the patients were estimated to be high-risk based on an ASA score of 3 or higher. Consequently, we performed surgeries under local anesthesia with proper sedation management in cooperation with the anesthetist in these 3 patients. Preoperatively, the patients were placed in the prone position on a radiolucent table with head immobilization in a carbon Mayfield clamp. The optimal position of the cervical spine depended on preoperative reduction. Subsequently, the skin entry point was marked using C-arm fluoroscopy to locate the surgical sites of the $\mathrm{C} 1$ lateral mass and $\mathrm{C} 2$ pedicle.

To insert the $\mathrm{C} 1$ lateral mass screw (LMS), an initial 8-mm incision was made at the level of the atlas above the lateral mass. To avoid neurovascular injury during the puncture process, the blunt end of a $2.0-\mathrm{mm}$ Kirschner wire (K-wire) was used and adjusted to reach the posterior surface of the $\mathrm{C} 1$ lateral mass under $\mathrm{C}$-arm fluoroscopic guidance (Fig. 1A). When an acceptable site was reached, a primary dilator was rotated carefully into the bone surface to avoid neurovascular tissue, and then the K-wire was pulled out, turned around and inserted into the lateral mass as a guide wire. Next, a working sheath was inserted along the guide wire (Fig. 1B). Subsequently, the remaining processes were performed under endoscopy in the same manner as in percutaneous endoscopic surgery.

Clear visualization was achieved with saline perfusion. Dissection and coagulation were performed 
using low-energy radiofrequency to precisely identify the structures under a visual field magnified 10 to 20 times. We could clearly identify the $C 2$ nerve root, lateral mass, and posterior arch (Fig. 2A1), and we could observe the paravertebral pulse from the VA (Supplemental Video). We selected the middle point of the interface of the inferior rim of the posterior arch and the posterior surface of the lateral mass as the screw entry point (13) (Fig. 2A2). After verifying the entry point under endoscopy, a cortex window was drilled using a high-speed burr. Occasionally, a small portion of the inferior aspect of the posterior arch was removed to facilitate screw placement. Then, an awl was used to create a screw path aiming at the lateral mass center with a cephalad angulation of $20^{\circ}$ (13). After detecting the intactness of the screw path using a blunt probe and tapping with the relevant instrument (Fig. 1C), a guide wire was inserted into the pilot hole. Then, sequential dilating tubes were inserted along the guide wire, and the incision was extended to approximately $16 \mathrm{~mm}$ (Fig. 3A). The last tube with a 16-mm inner diameter remained in place to serve as a working channel. After verifying the pilot hole and the screw insertion direction on the same axis as the working channel under endoscopy, we removed the endoscope and guide wire. Then, a suitable screw was inserted along the working channel that was kept in the fixed axis (Fig. 3B,C). Using fluoroscopic assistance, the lateral trajectory of the screw point lay in the direction of the anterior C1 tubercle (Fig. 1D).

The same surgical technique was used for the $\mathrm{C} 2$ pedicle screw (PS). To prevent screw malposition, the medial border of the $\mathrm{C} 2$ pedicle was first identified using a right-angle detector to detect the border of the ligamentum flavum and lamina. The entry point was selected approximately $5 \mathrm{~mm}$ inferior to the superior border of the C2 lamina and $7 \mathrm{~mm}$ lateral to the lateral border of the spinal canal (Fig. 2C,D). The screw path was selected approximately $20^{\circ}$ to $30^{\circ}$ in medial and cephalad angulation toward the anterior edge of the C1-C2 joint under lateral fluoroscopic guidance (14) (Fig. 1E). After placing 2 screws, a rod was inserted through the caudal incision beyond the fascia and moved rostrally by gently pushing the muscles (Figs. 1F, 3D). Rod bending was performed depending on the position of reduction under lateral fluoroscopy. Then, the rod was fixed with blocker screws (Figs. 1G,H). Eventually, the 2 incisions were sutured with one stitch each, with a drainage tube remaining under the deep fascia (Supplemental Video).

In all patients, minimally invasive spine instruments, a screw-rod system (WEGO Co., Weihai, Shandong, China) with polyaxial screws composed of titanium with 3.5- to 4.5-

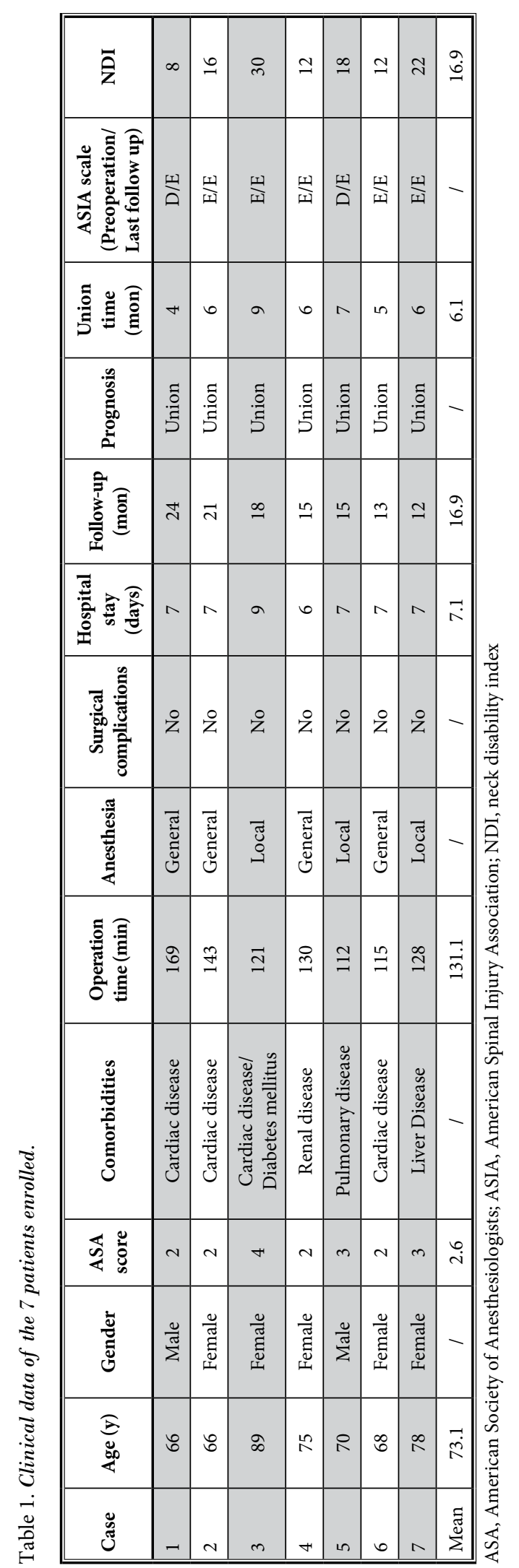



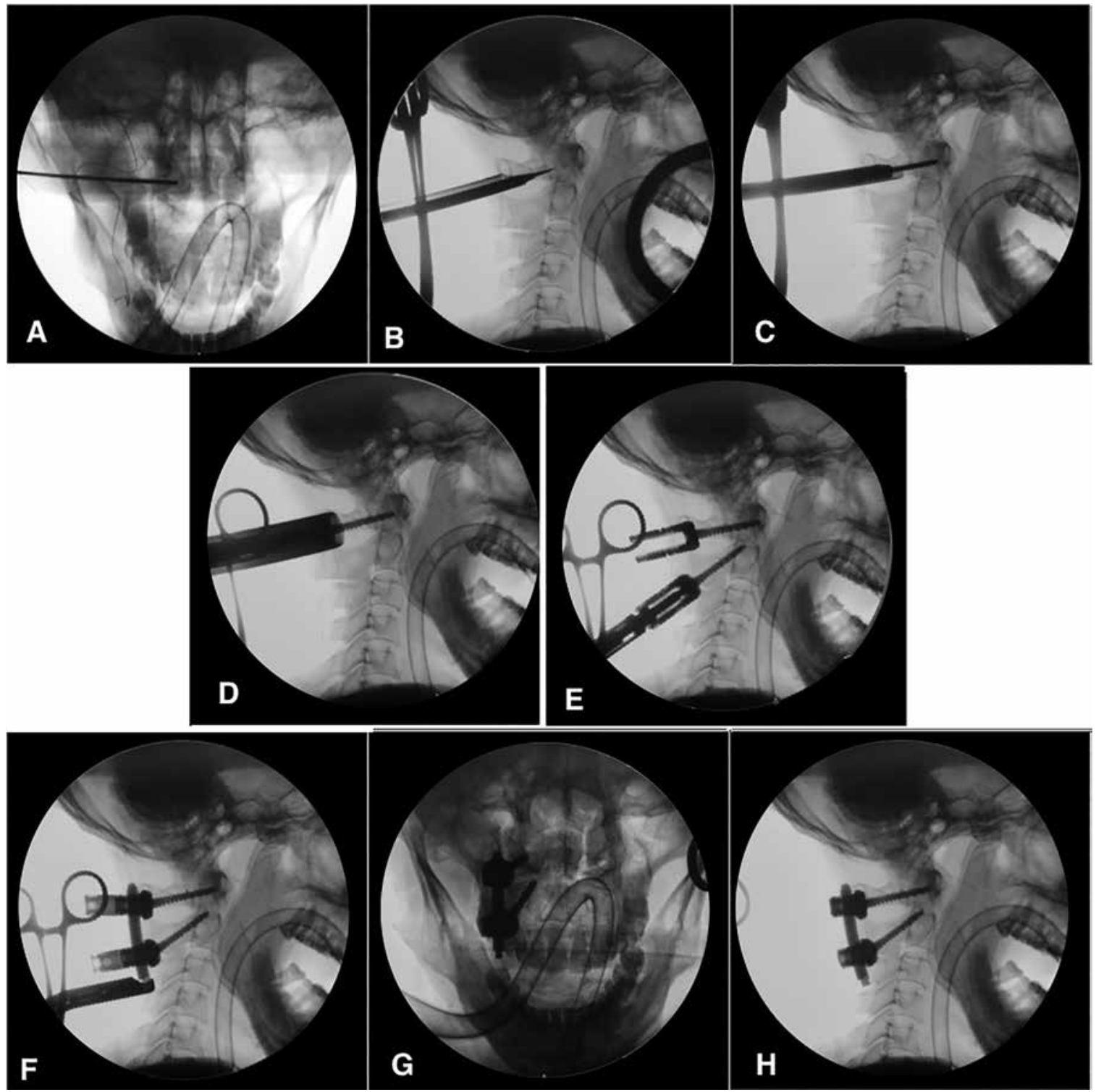

Fig. 1. Intraoperative $x$-ray film. A, anterior-posterior ( $A-P)$ view showing a blunt $K$-wire on the posterior surface of a $C 1$ lateral mass. $B$, Lateral view showing the primary dilator and working sheath inserted along the guide wire. $C$, Tapping the pilot screw hole. D, Placing the C1 lateral mass screw. E, Placing the C2 pedicle screw. F, Percutaneously inserting the rod. G, A-P view of the postoperative film. H, Lateral view of the postoperative film.

mm diameters and a connecting rod (3.2-mm titanium) were used for surgery.

\section{Postoperative Management}

Intraoperative data on operative time, blood loss volume, and surgery-related complications were recorded. Thin-cut CT and x-ray were performed postop- eratively in all patients (Fig. 4). Intravenous antibiotics were administered, and a drainage tube was left for 24 hours postoperatively if no complications occurred. Then, patients were allowed to walk and sit with immobilization in a rigid neck collar if their condition permitted. Patients were required to wear the neck collar for approximately 12 weeks after surgery. Routine 
Fig. 2. Schematic figures of screws entry points. A1, Endoscopic structures displayed around the C1 lateral mass screw (LMS) entry point. $A 2,3 D$ reconstruction of C1, where the black point represents the entry point of the $C 1$ lateral mass screw and the red circle represents the area of the endoscopic visual field. B1, Endoscopic structures displayed around the C2 pedicle screw (PS) entry point. $B 2,3 D$ reconstruction of C2, where the black point represents the entry point of the $C 2$ $P S$ and the red circle represents the area of the endoscopic visual field.

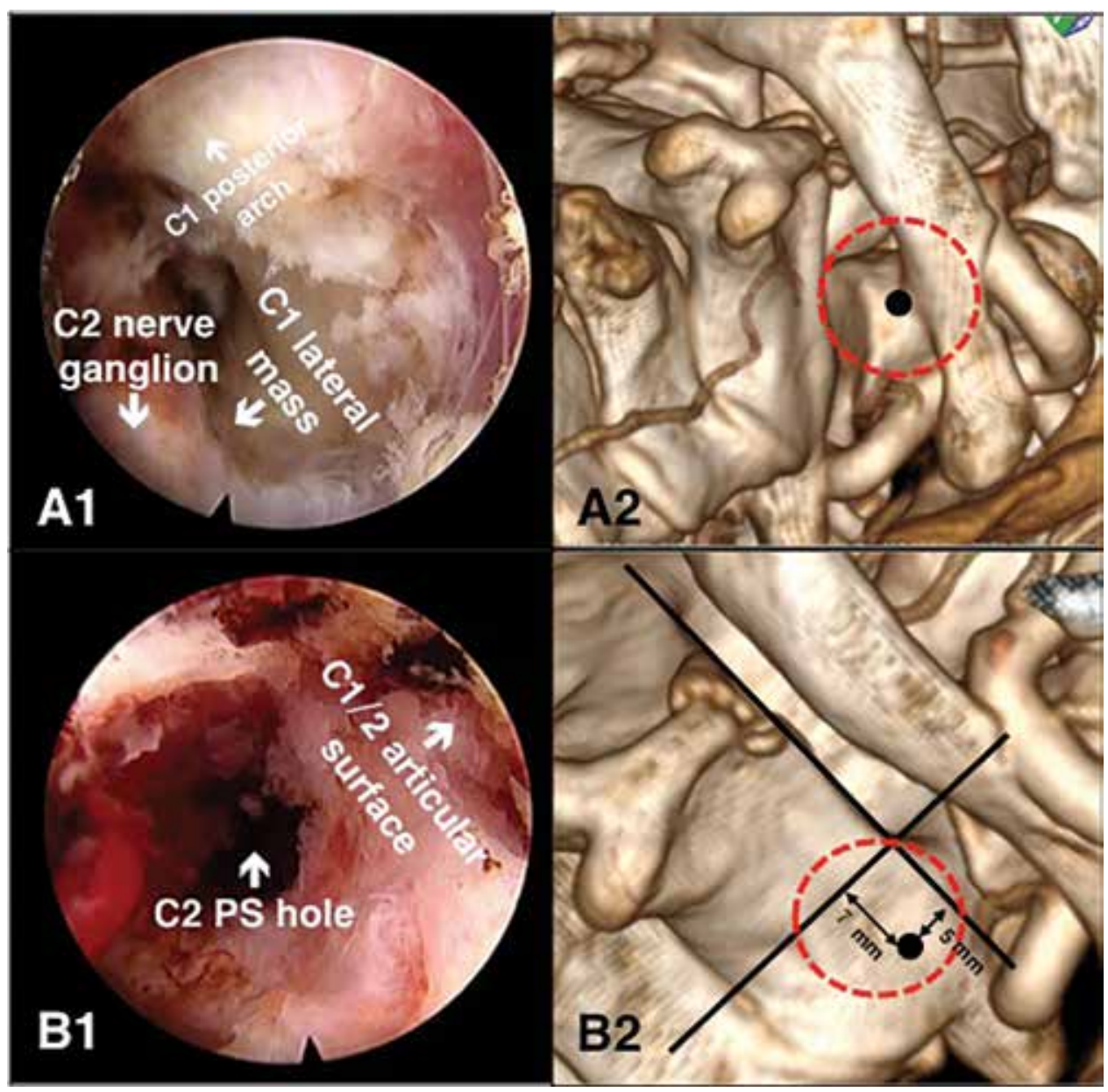

follow-ups were performed at 6 weeks and 3, 6, 9, 12, and 24 months postoperatively. The prognosis of bone union time, American Spinal Injury Association (ASIA) scale scores, Neck Disability Index (NDI) scores, and postoperative complications were collected for assessment. When fracture union was observed on CT examination, the patient was advised to undergo percutaneous endoscopically-assisted surgery for internal implant removal to retain atlantoaxial movement (Fig. 5).

\section{Ethical Considerations}

We received permission from the ethics committee of our hospital and written informed consent from all patients.

\section{Results}

The surgical goal was successfully achieved in all patients, 3 of whom had high ASA scores $(\geq 3)$ and underwent surgery under local anesthesia. The operative time ranged from 112 to 169 minutes (mean, 131.1 minutes). The total blood loss volume was less than 50 $\mathrm{mL}$. No neurovascular complications were observed intraoperatively or postoperatively. All patients rapidly returned to their prior level of function and were followed up for 12 to 24 months (average, 16.9 months). Bone union was achieved in all patients, and the average union time was 6.1 months. Other clinical data, including ASIA scale and NDI scores, are presented in Table 1. No screw loosening or breakage occurred in any of the patients.

\section{Discussion}

In recent decades, the optimal treatment for type II geriatric odontoid fractures has remained controversial. Optimal treatment of geriatric patients with type II odontoid fractures would help them return to 


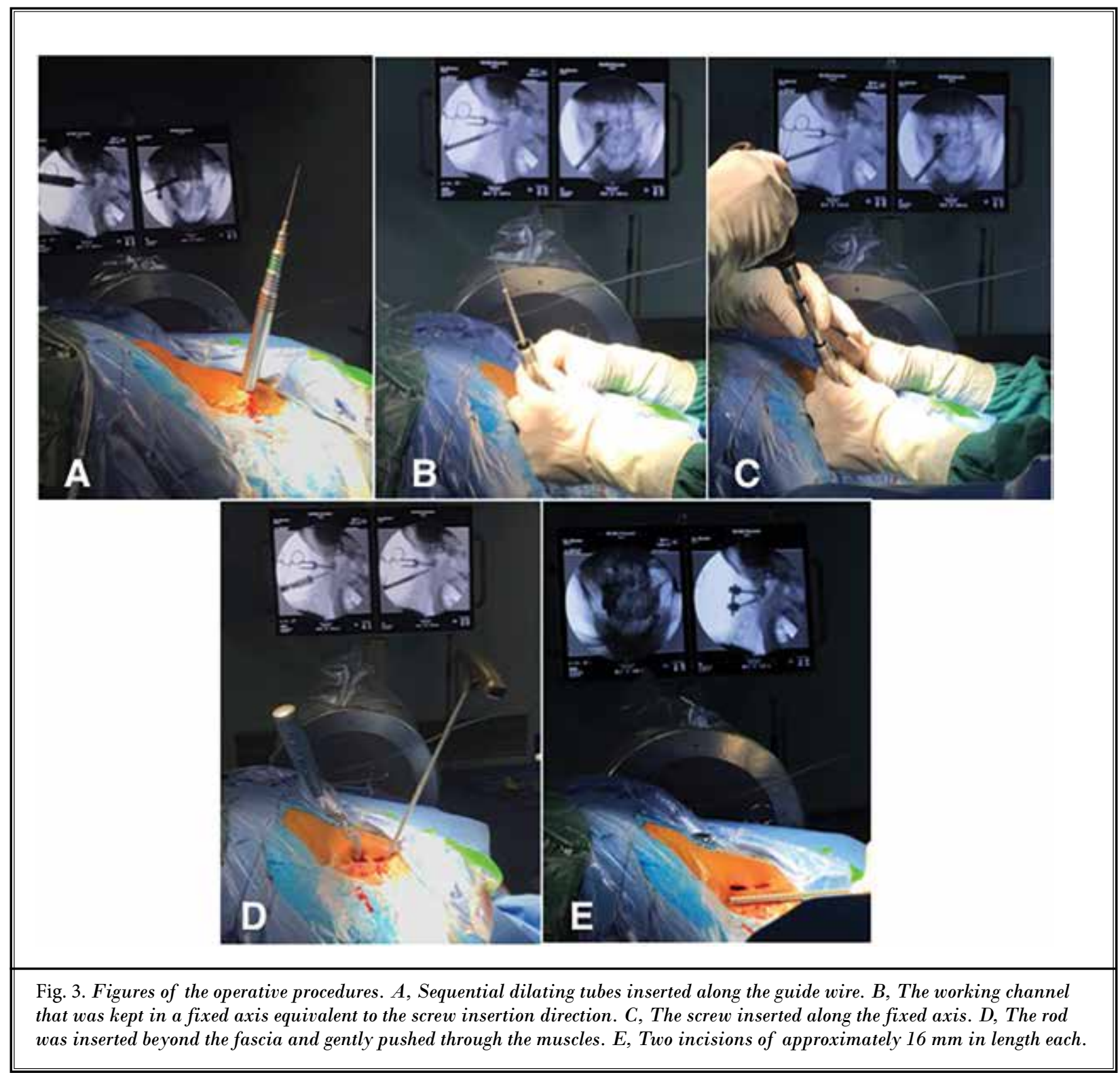

their prior level of function as rapidly as possible while avoiding the morbidity and mortality associated with prolonged and bedbound hospitalization (7). The current view suggests that for geriatric patients sustaining these fractures who are able to undergo general anesthesia, the benefits of surgery outweigh the associated risks (10). However, an increased ASA score predicted significant increases in complication rates for morbidity and mortality postoperatively (12). Consequently, an optimal treatment that avoids the disadvantages of non-operative management and decreases the surgi- cal risk is needed for patients with increased risk from anesthesia.

Posterior C1-C2 fusion is the most common operation for type II odontoid fractures. This procedure is performed via posterior open surgery and has been shown to yield a good fusion rate and clinical outcome. However, posterior $\mathrm{C} 1-\mathrm{C} 2$ fusion has the innate disadvantages of considerable soft tissue invasion, blood loss, risk of neurovascular injury (15), and loss of atlantoaxial motion $(16,17)$. Therefore, in recent years, posterior $\mathrm{C} 1-\mathrm{C} 2$ nonfusion fixation has been progres- 
Fig. 4. Preoperative and postoperative computed tomography (CT) reconstructions. $A$, Preoperative sagittal image. $B$, Preoperative coronal image. C, Postoperative sagittal image of screws. D, Postoperative transverse image of the C1 lateral mass screw. E, Postoperative transverse image of the C2 pedicle screw.

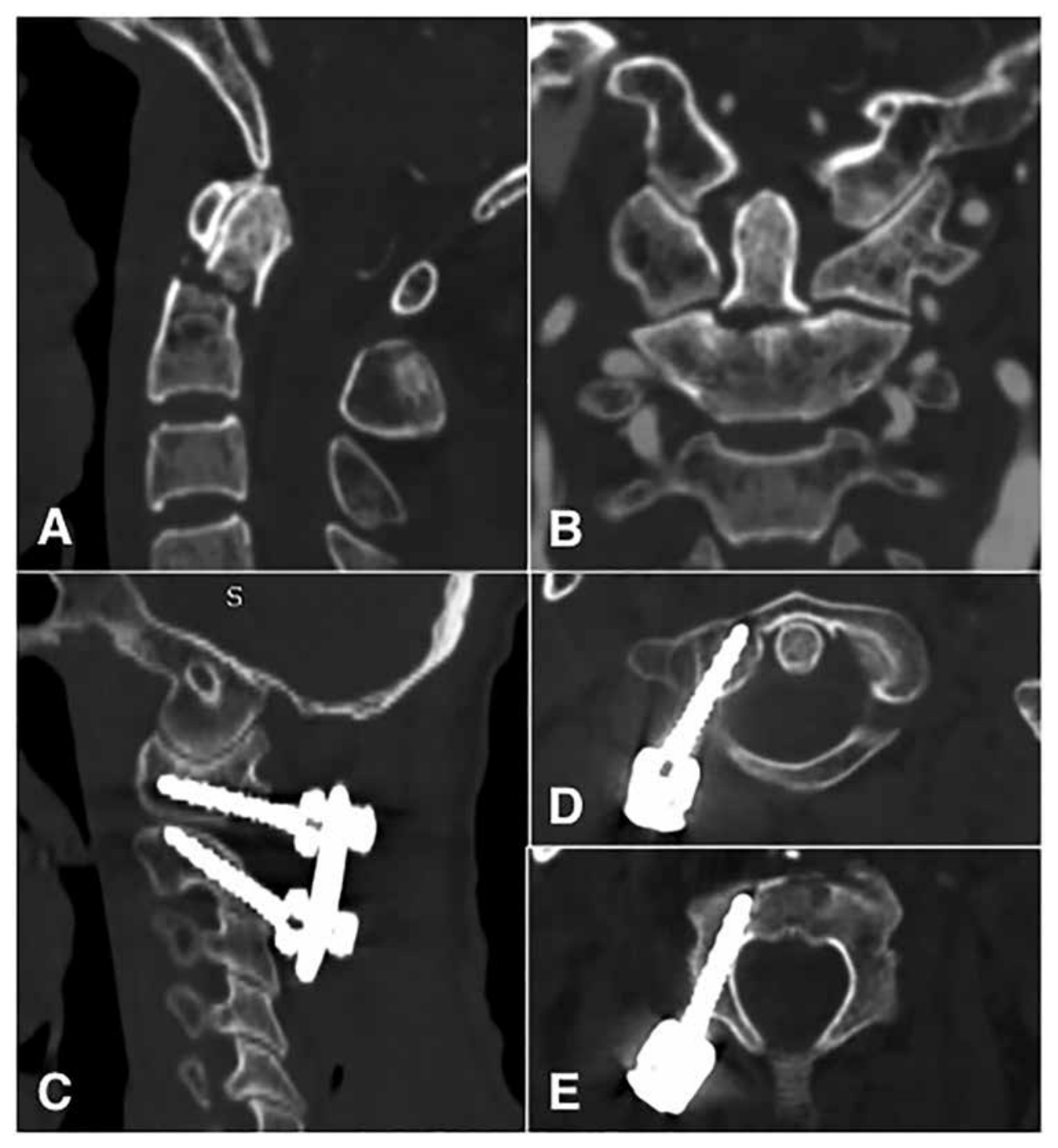

sively applied to treat odontoid fractures with an intact transverse ligament. Posterior temporary fixation can retain the motion of $\mathrm{C} 1-\mathrm{C} 2$ and achieve similarly good clinical outcomes as with anterior screw fixation in the treatment of type II odontoid fractures (16). Overall, most authors suggest that the anterior odontoid approach should primarily be considered in younger patients with good bone quality, amenable fracture obliquity, and an appropriate body habitus (10). In addition, anterior retropharyngeal approaches pose a risk of severe complications, such as spinal cord injury, esophageal and pharyngeal perforation, arterial injury, and airway obstruction (18). Meanwhile, anterior odontoid screw fixation requires highly technical skills and is not suitable for special fracture types that extend from anterior-inferior to posterior-superior or for obviously comminuted fractures (19). For the above reasons, we ultimately selected posterior nonfusion fixation as the primary surgical strategy.

Recently, percutaneous PS placement using minimally invasive instruments has been shown to decrease blood loss, reduce soft tissue invasion, and minimize postoperative pain in the thoracic and lumbar spine (20). A few recent articles have reported the percutaneous screw technique in the cervical spine, which was performed under 3-dimensional fluoroscopic guidance with tubular dilator retractors in all cases (21-23). Threedimensional navigation can help doctors perform sur- 


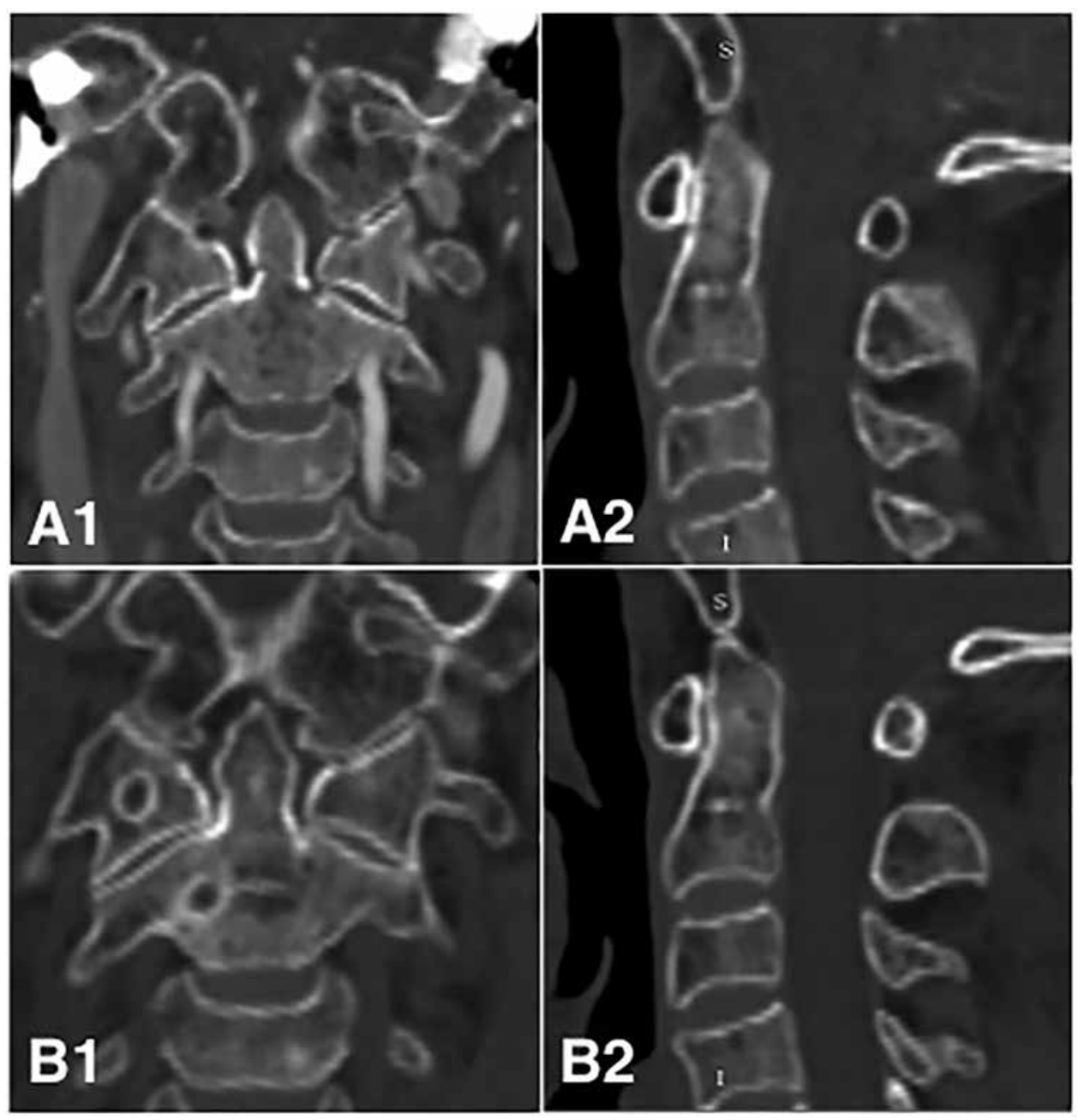

Fig. 5. Computed tomography (CT) reconstruction of pre-operation and after internal fixation removal. A1, Preoperative sagittal image. A2, Preoperative coronal image. B1, Sagittal image after internal fixation removal. B2, Coronal image after internal fixation removal.

gery efficiently and safely, reducing the risk and complications of spinal surgery. Due to the high cost of the equipment and high technical requirements, only a few large hospitals currently own such equipment, which is a main limitation of clinical application. In recent years, the percutaneous endoscopic technique has gradually received approval from spinal surgeons. Compared with traditional surgery, the percutaneous endoscopic technique has the main advantages of clear visualization of anatomical structures, a rapid postoperative recovery, and less damage to soft tissues. To the best of our knowledge, percutaneous atlantoaxial screw-rod fixation has not been previously reported. Performing percutaneous procedures only relying on fluoroscopy is technically challenging. Therefore, assistance from percutaneous endoscopy to achieve percutaneous atlantoaxial screw-rod fixation is reasonable, and the clear visualization of anatomical structures under endoscope will provide significant help for screws placement.

To achieve the goal of permitting patients to return to their prior level of function as rapidly as possible while avoiding the morbidity and mortality associated with prolonged inactivity and hospitalization (7), unilateral screw-rod fixation was performed as a limited means of internal fixation with less invasiveness and a reduced operative time. Meanwhile, the additional use 
of a rigid cervical collar postoperatively may improve the fracture union rate.

Generally, a C1 LMS poses risks of injuring the paravertebral venous plexus and the C2 nerve root. Occasionally, injury of the venous plexus may result in persistent venous bleeding, and nerve injury may present as nerve dysfunction in the distribution of the $\mathrm{C2}$ nerve, including neuralgia, numbness, dysesthesia, and paresthesia. Therefore, we used the blunt side of a Kwire to locate the lateral mass to avoid C2 nerve root injury. Furthermore, continuous perfusion of saline under percutaneous endoscopy can generate hydrostatic pressure to stop venous bleeding. Meanwhile, coagulation is performed using low-energy radiofrequency to maintain a clear visual field to precisely identify structures under endoscopy. Therefore, blood loss is reduced, and the magnifying effect of the endoscope helps to precisely identify the screw entry point to ensure the screw's appropriate placement.

Although we successfully treated a few patients with type II odontoid fractures using this technique, we need to further follow the patients' clinical outcomes, especially complications, the NDI, the union rate, and mortality, to comprehensively assess this novel technique. Additionally, knowing whether internal fixation devices should be retained or removed in these geriatric patients requires long-term clinical observation.

\section{Conclusion}

Endoscopically-assisted percutaneous unilateral atlantoaxial screw-rod nonfusion fixation is a feasible technique for type II odontoid fractures in geriatric patients. This method offers a compromise between non-operative and operative treatment and helps such patients to rapidly return to their prior level of function.

Funding: The study was supported by the Special Foundation for Social Safeguard and Scientific Innovation of Chongqing (No. cstc2016shms-ztzx10001-6), Chongqing Research and Innovation project of graduate students (No. YB17112), the key project of Chongqing Science and Technology Commission (No. cstc2019jscx-gksb0014), and the key project of Chongqing Health Commission (No.2016ZDXM007).

\section{References}

1. Schroeder GD, Kepler CK, Kurd MF, et al. A systematic review of the treatment of geriatric type II odontoid fractures. Neurosurgery 2015; 77:S6-S14.

2. Ryan MD, Taylor TK. Odontoid fractures in the elderly. J Spinal Disord 1993; 6:397-401.

3. Ryan MD, Henderson JJ. The epidemiology of fractures and fracture-dislocations of the cervical spine. Injury 1992; 23:38-40.

4. Anderson LD, D'Alonzo RT. Fractures of the odontoid process of the axis. J Bone Joint Surg Am 1974; 56:1663-1674.

5. Muller EJ, Wick M, Russe O, Muhr G. Management of odontoid fractures in the elderly. Eur Spine J 1999; 8:360-365.

6. Koech F, Ackland HM, Varma DK, Williamson OD, Malham GM. Nonoperative management of type II odontoid fractures in the elderly. Spine 2008; 33:2881-2886.

7. Vaccaro AR, Kepler CK, Kopjar B, et al. Functional and quality-of-life outcomes in geriatric patients with type-II dens fracture. J Bone Joint Surg Am 2013; 95:729-735.

8. Butler JS, Dolan RT, Burbridge M, et al. The long-term functional outcome of type II odontoid fractures managed non-operatively. Eur Spine J 2010; 19:1635-1642.

9. Evaniew N, Yarascavitch B, Madden K, et al. Atlantoaxial instability in acute odontoid fractures is associated with nonunion and mortality [published online ahead of print March 21, 2014]. Spine J 2015; 15:910-917.

10. Wagner SC, Schroeder GD, Kepler CK, et al. Controversies in the management of geriatric odontoid fractures. J Orthop Trauma 2017; 31:S44-S48.

11. Chapman J, Smith JS, Kopjar B, et al. The AOSpine North America Geriatric Odontoid Fracture Mortality Study: A retrospective review of mortality outcomes for operative versus nonoperative treatment of 322 patients with long-term follow-up. Spine (Phila Pa 1976) 2013; 38:1098-1104.

12. Hackett NJ, De Oliveira GS, Jain UK, Kim JYS. ASA class is a reliable independent predictor of medical complications and mortality following surgery. Int J Surg 2015; 18:184-190.

13. Fensky F, Kueny RA, Sellenschloh K, et al. Biomechanical advantage of $\mathrm{C}_{1}$ ped- icle screws over $C_{1}$ lateral mass screws: A cadaveric study. Eur Spine J 2014; 23:724-731.

14. Ebraheim N, Rollins JR Jr, Xu R, Jackson WT. Anatomic consideration of $\mathrm{C}_{2}$ pedicle screw placement. Spine (Phila Pa 1976) 1996; 21:691-695.

15. Wu AM, Jin HM, Lin ZK, Chi YL, Wang $X Y$. Percutaneous anterior $\mathrm{Cl}_{1} / 2$ transarticular screw fixation: Salvage of failed percutaneous odontoid screw fixation for odontoid fracture. J Orthop Surg Res 2017; 12:141.

16. Guo Q, Zhang M, Wang L, Lu X, Guo $X, N i$ B. Comparison of atlantoaxial rotation and functional outcomes of two nonfusion techniques in the treatment of Anderson-D'Alonzo type II odontoid fractures. Spine 2016; 41:E751-E758.

17. Guo Q, Deng Y, Wang J, et al. Comparison of clinical outcomes of posterior $\mathrm{C}_{1}-\mathrm{C}_{2}$ temporary fixation without fusion and $\mathrm{C}_{1}-\mathrm{C}_{2}$ fusion for fresh odontoid fractures. Neurosurgery 2016; 78:77-83.

18. Lin B, Lu C, Yu H, Zhang W, Yang W. Comparison of microendoscopic discectomy system and anterior open approach in treatment of unstable odon- 
toid fracture with cannulated screw internal fixation. Acta Orthop Belg 2014; 80:529-536.

19. Grauer JN, Shafi B, Hilibrand AS, et al. Proposal of a modified, treatment-oriented classification of odontoid fractures. Spine J 2005; 5:123-129.

20. Mobbs RJ, Sivabalan P, Li J. Technique, challenges and indications for percuta- neous pedicle screw fixation. ] Clin $\mathrm{Neu}$ rosci 2011; 18:741-749.

21. Kantelhardt SR, Keric N, Conrad J, Archavlis E, Giese A. Minimally invasive instrumentation of uncomplicated cervical fractures. Eur Spine J 2016; 25:127-133.

22. Buchholz AL, Morgan SL, Robinson LC, Frankel BM. Minimally invasive per- cutaneous screw fixation of traumatic spondylolisthesis of the axis. J Neurosurg Spine 2015; 22:459-465.

23. Wang MY, Levi ADO. Minimally invasive lateral mass screw fixation in the cervical spine: Initial clinical experience with long-term follow-up. Neurosurgery 2006; 58:907-912. 